\title{
Ground state properties of the Holstein model near the adiabatic limit
}

\author{
Zhou Li, D. Baillie ${ }^{1}$, C. Blois ${ }^{2}$, F. Marsiglio \\ Department of Physics, University of Alberta, Edmonton, Alberta, Canada, T6G 2G7
}

(Dated: November 6, 2018)

\begin{abstract}
We adapt a variational procedure to calculate ground state properties of the Holstein model in the adiabatic limit. At strong coupling, this adaption leads to rapid convergence of results. The intermediate coupling regime is further handled with an adaptive algorithm. We also use semiclassically derived results for the adiabatic end-point, along with weak coupling perturbation theory. These establish weak and strong coupling (or large and small polaron, respectively) regimes in two dimensions or higher. As is well known, these are connected smoothly, but the cross-over becomes increasingly abrupt as the phonon frequency decreases.
\end{abstract}

PACS numbers:

\section{INTRODUCTION}

There has been considerable work performed over the last two decades on the Holstein model $\stackrel{1}{ }$. Interest in this model is fueled by the fact that it serves as the paradigm for electron-phonon interactions, much like the Hubbard model $^{2}$ serves the same purpose for electron-electron interactions. While a considerable amount of this work has focussed on the many-electron problem, another subset has examined the single-electron, or polaron problem. A recent review of this work is available, for example, in Ref. [3, 4].

In our opinion, the most promising numerical technique for determining polaron properties in the thermodynamic limit is the variational procedure outlined by Trugman and coworkers ${ }^{5,6}$. With this method properties such as the ground state energy and the effective mass are readily obtained, in any dimension, over almost all parameter regimes. One range of parameter space that has remained difficult, however, is near the adiabatic limit, which is what we address in this paper. The actual adiabatic limit was first treated by Kabanov and Mashtakov ${ }^{7}$; they found that in one dimension (1D), the electron retains polaronic character for all electron-phonon coupling strengths, while in two dimensions and higher there is a critical coupling strength, below which the electron behaves in a 'free-electron-like' manner, and above which it is polaronic. At the same time, away from the adiabatic limit the problem is known from numerical solutions to have a smooth crossover as a function of coupling strength (i.e. no abrupt transition), so it is of interest to pursue this crossover as the phonon frequency decreases towards zero. This was done to some degree in Refs. 8,9, but only for rather small lattices in one dimension. Our aim is to examine this limit using the Trugman variational technique ${ }^{6,10}$.

The outline of the paper is as follows. In the next section we outline the model, and establish notation, etc. In Section III we describe some refinements to the variational method, and provide some illustrative examples to demonstrate the improvement in convergence. In Section IV we provide some numerical results as the adiabatic parameter $\omega_{E} / t$ approaches zero. Also provided are some perturbation theory results $\frac{9}{}$, which can be reinterpreted to provide constraints for the numerical results. In Section $\mathrm{V}$ we show some results concerning the expected numbers of phonons in the ground state, which gives another indication of the difficulty of the adiabatic limit. Finally, we close with a summary.

\section{THE MODEL}

The model that most simply describes an electron interacting with optical phonons is the Holstein Hamiltonian, given by

$$
\begin{aligned}
H & =-t \sum_{i, \delta}\left(c_{i}^{\dagger} c_{i+\delta}+c_{i+\delta}^{\dagger} c_{i}\right) \\
& +\sum_{i}\left[\frac{p_{i}^{2}}{2 M}+\frac{1}{2} M \omega_{E}^{2} x_{i}^{2}\right]-\alpha \sum_{i} n_{i} x_{i}
\end{aligned}
$$

which is also written

$$
\begin{aligned}
H & =-t \sum_{i, \delta}\left(c_{i}^{\dagger} c_{i+\delta}+c_{i+\delta}^{\dagger} c_{i}\right)-g \omega_{E} \sum_{i} n_{i}\left(a_{i}+a_{i}^{\dagger}\right) \\
& +\omega_{E} \sum_{i} a_{i}^{\dagger} a_{i}
\end{aligned}
$$

where $c_{i}^{\dagger}\left(c_{i}\right)$ creates (annihilates) an electron at site $i$ (the spin label is suppressed) and $n_{i} \equiv c_{i}^{\dagger} c_{i}$ is the number density operator. The ion momentum $p_{i}$, and displacement $x_{i}$ are quantized via

$$
\begin{aligned}
& x_{i} \equiv \sqrt{\frac{1}{2 M \omega_{E}}}\left(a_{i}^{\dagger}+a_{i}\right) \\
& p_{i} \equiv i \sqrt{\frac{M \omega_{E}}{2}}\left(a_{i}^{\dagger}-a_{i}\right),
\end{aligned}
$$

where $M$ is the ion mass (we set $\hbar \equiv 1$ ) and $a_{i}^{\dagger}\left(a_{i}\right)$ creates (annihilates) a phonon at site $i$. The sum over $i$ is over all sites in the lattice, whereas the sum over $\delta$ is over nearest neighbors. Here, as the notation already suggests, we confine ourselves to nearest neighbor hopping only. The parameters are the hopping integral $t$, the phonon 
frequency $\omega_{E}$, and the coupling of the electron to the oscillator degrees of freedom, $\alpha$. This parameter is the bare coupling between the electron and the ion; however, it is rarely used, and instead in the polaron literature the dimensionless coupling constant $g \equiv \frac{1}{\omega_{E}} \frac{\alpha}{\sqrt{2 M \omega_{E}}}$ is used. In the many-body literature, the dimensionless parameter $\lambda \equiv \frac{2 \omega_{E} g^{2}}{W} \equiv \frac{\alpha^{2}}{M \omega_{E}^{2} W}$ is used, where $W \equiv 2 z t$ is the electronic bandwidth for a cubic tight-binding model with coordination number $z(z=2,4,6$ in $1,2,3$ dimensions, respectively). The parameter $\lambda$ has historical significance for the effective mass of degenerate electrons weakly coupled to phonons. Alternatively, and most useful in the strong coupling regime, the parameter $g$ (or $g^{2}$ ) was used in the Lang-Firsov transformation $\frac{11}{1}$, and leads to a band narrowing factor $t \rightarrow t^{*}=t e^{-g^{2}}$ in first order degenerate perturbation theory. We write all energy scales in terms of the hopping integral, $t$, which, hereafter is set to unity. So two dimensionless parameters which usefully characterize this problem ${ }^{3}$ are $\omega_{E} / t$, the adiabaticity parameter, and, $\lambda \equiv 2 g^{2} \omega_{E} / W$. Actually, as we argue below in Section III, in two dimensions it is arguably more useful to use $\lambda \equiv 2 g^{2} \omega_{E} /(4 \pi t)=g^{2} \omega_{E} /(2 \pi t)$, where $1 /(4 \pi t)$ is the value of the non-interacting electron density of states at the bottom of the band (as opposed to the average density of states).

As mentioned in the introduction this model has been most successfully analyzed using a refinement of the standard Lanczos method due to Trugman ${ }^{5,6}$. Very accurate results can be obtained in any dimension 10 in almost all parameter regimes $\stackrel{3,6,10}{*}$. A difficulty remains for moderately to strongly coupled systems with low adiabaticity parameter $\omega_{E} / t$. For example, if one uses the Lang-Firsov transformation 11 to define the zeroth order strongly coupled wave function, then the average number of phonons in the ground state can be readily determined to be approximately $g^{2}$. For typical parameters in the moderately coupled regime (in one dimension), say $\omega_{E}=0.05 t$, and $\lambda=1.0$, then $g^{2}=40$, and this is the approximate number of phonons in the ground state. The Trugman procedure starts with a bare electron; on a moderate work station a feasible number of applications of the Hamiltonian is $N_{h}=22$ (as remarked in Ref. 6), which produces a Hilbert space of order $10^{7}$. This process with $N_{h}=22$ produces states that contain a maximum of 22 phonons, and cannot possibly yield the correct ground state.

\section{REFINEMENT OF THE TRUGMAN METHOD}

We have examined two simple refinements to the Trugman method $\frac{12}{2}$; instead of starting with the bare electron state (properly extended throughout an infinite lattice), we first start with the state which is used as the unper- turbed state in the strong-coupling limit $\stackrel{9,11}{ }$ :

$$
|\psi\rangle=e^{-g^{2} / 2} \sum_{\ell} e^{i k R_{i}} e^{-g \hat{a}_{\ell}^{\dagger}} \hat{c}_{\ell}^{\dagger}|0\rangle
$$

where the sum is over all lattice sites $\frac{13,14}{}$. As we shall see in what follows this speeds up convergence considerably in the strong coupling regime (either $\lambda>>1$ or $\omega_{E}<<$ $t$ ). An example of the increased convergence is illustrated in Fig. 1. Following Fig. 2 of Ref. 10 we show the fractional error $\Delta_{N} \equiv\left|\left(E_{N}-E_{N-1}\right) / E_{N}\right|$ as a function of the number of states kept in the Hilbert space, for two sets of parameters, both of which have $g^{2}=20$, using the bare electron as the starting state and using the strongcoupling solution as the starting state. There is a clear numerical advantage to using the latter. In Fig. 1(c) we show the fractional error for a parameter regime near $\lambda \approx 1$, where the strong coupling start is better even for values of $\lambda<1$. It is also clear that as $\lambda$ increases beyond the range of this figure, the refinement becomes increasingly useful.

In pursuit of more severe disparate electron and phonon energy scales we found that even starting with the strong coupling solution resulted in slow convergence when $\lambda$ was of order unity. A remedy to this difficulty is the following procedure: start at large values of $\lambda$, where convergence is readily obtained after a few iterations. Lower the value of $\lambda$ by a small amount, and use as a starting wave function the previous solution, truncated to include components with some minimal amplitude (so that a few hundred basis states at most are used). Then converge the solution for this value of $\lambda$, lower it, and continue the process until the desired range is covered. We have found this latter procedure to be the most robust, particularly when the phonon frequency is much smaller than the electron hopping parameter, $t$.

In Fig. 2 we show the ground state energy $E_{0}$ vs. $\lambda$ for various values of the phonon frequency; this is in one dimension. Fig. 3 shows similar results in two dimensions. It is clear that as the phonon frequency decreases, the crossover region near $\lambda \approx 1$ (actually, the "critical' value of $\lambda$, only valid in the adiabatic limit, is closer to 0.55 ) becomes sharper. This is consistent with the result that, in the adiabatic limit, there is a transition from a small polaron state to a free electron-like state, in dimensions two and higher ${ }^{7}$. Nonetheless, as is known through other considerations $\frac{15}{}$, for any non-zero phonon frequency, the crossover is smooth.

To summarize this section, we have obtained numerically exact results for a wide range of parameters, by using refinements to that used in Ref. 5, 6. In particular, we obtain well converged results over all coupling strengths and for low phonon frequencies, $\omega_{E}<<t$. The results for low frequencies in particular illustrate a rather abrupt crossover to a regime where multi-phonon processes are prevalent. To what extent they play a crucial role even at intermediate coupling strengths is the subject of the next section. 

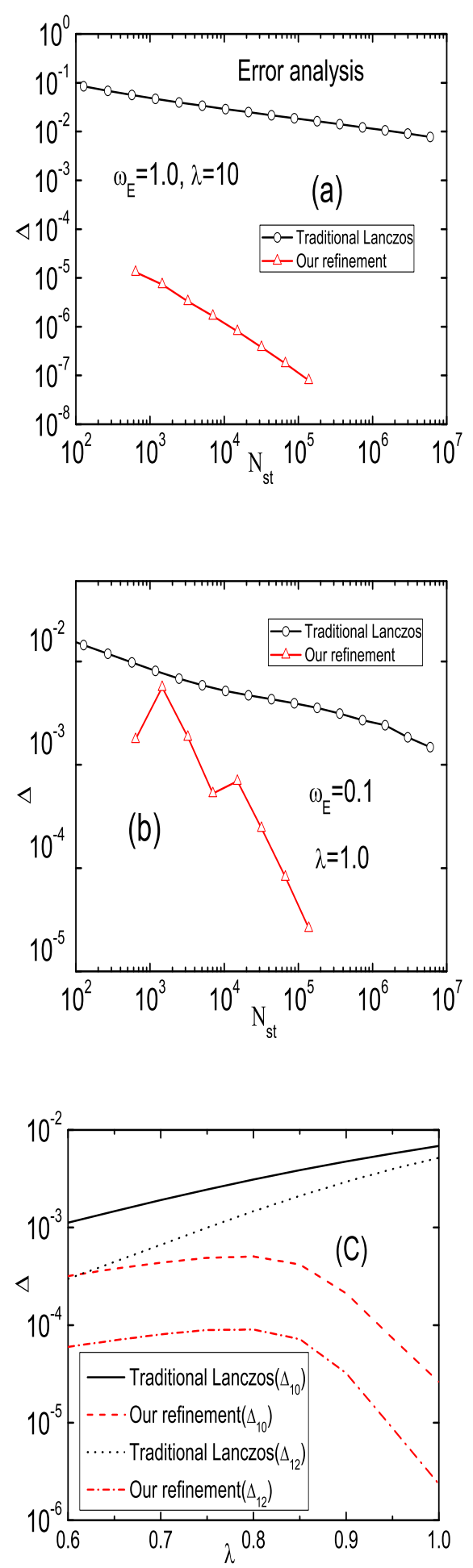

FIG. 1: (color online) Fractional error vs. number of states for (a) a very strong coupling case, and (b) a moderate coupling, but with $\omega_{E} / t=0.1$. In both cases our refinement speeds up convergence considerably. In (c) we show the improvement over a range of coupling strength near $\lambda=1$; for $\lambda>1$ (not shown) it is clear that the refinement leads to much better convergence. Note that the fractional error does not have to decrease monotonically with the number of states added.


FIG. 2: (color online) Ground state energy $E_{0}$ vs. $\lambda$ and $m^{*} / m$ vs. $\lambda$ for various phonon frequencies, in one dimension. There really is no special value of $\lambda$ singled out in these curves, consistent with the crossover phenomenon discussed in the text.

\section{PERTURBATION THEORY}

Perturbation theory can be performed both from the weak and the strong coupling limits. ${ }^{9}$ In that work we obtained, to second order (in $g$ ), in one dimension, the self energy

$$
\Sigma_{1 \mathrm{D}}(\omega+i \delta)=\frac{\lambda \omega_{E} \operatorname{sgn}\left(\omega-\omega_{E}\right)}{\sqrt{\left(\frac{\omega-\omega_{E}}{2 t}\right)^{2}-1}} ;
$$



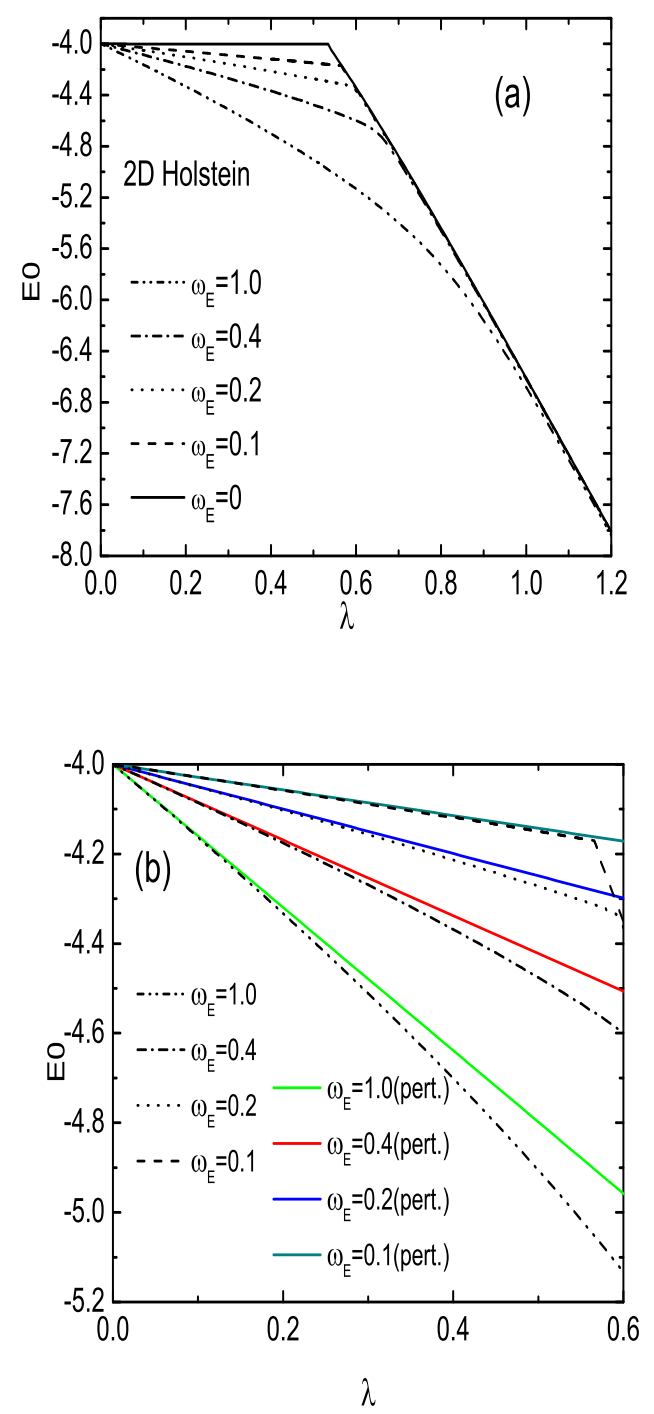

FIG. 3: (color online) (a) Ground state energy $E_{0}$ vs. $\lambda$ for various phonon frequencies, in two dimensions. In contrast to the 1D results in Fig. 2, a 'special' value of $\lambda$ is now apparent $-\lambda \approx 0.55$. However, for any non-zero phonon frequency the behaviour below and above this special value is smoothly connected. Only in the adiabatic limit does the behaviour change abruptly.

(b) Expansion of the weak coupling regime showing the numerical results along side the perturbation theory results. Agreement is very good.

which leads to a ground state energy:

$$
E_{0}=-2 t\left(1+\lambda \sqrt{\frac{\omega_{E}}{4 t+\omega_{E}}}\right) .
$$

This expression can be understood as follows: for very large frequency $\omega_{E}>>t$ there is a correction by a factor $1+\lambda$ reminiscent of the mass renormalization for the
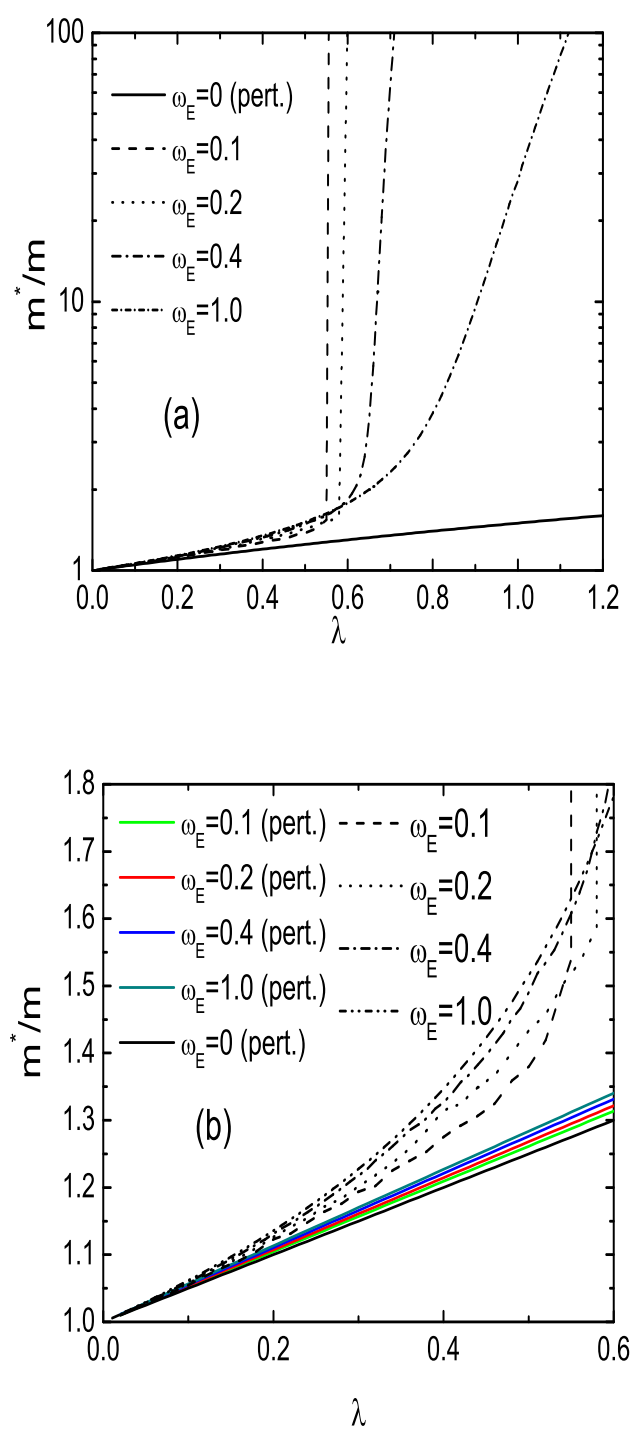

FIG. 4: (color online) Effective mass $m^{*} / m$ vs. $\lambda$ for various phonon frequencies, in two dimensions. Again, unlike the results in $1 \mathrm{D}$ in Fig. 2, a 'special' value of $\lambda$ is clear $\lambda \approx 0.55$. However, for any non-zero phonon frequency the behaviour below and above this special value is smoothly connected. Only in the adiabatic limit does the behaviour change abruptly.

(b) Expansion of the weak coupling regime showing the numerical results alongside the perturbation theory results. Agreement is not as good as in Fig. 3.

electron mass in a Fermi liquid state. On the other hand, as the frequency becomes small the first order correction vanishes. In fact, however, the most significant effect of the phonon coupling to a single electron occurs for low phonon frequencies, while the effect disappears for high phonon frequency. This is most readily seen by examin- 
ing the quasi-particle residue $z_{0}{ }^{\underline{9}}$

$$
z_{0}=1-\frac{\lambda}{2} \sqrt{\frac{t}{\omega_{E}}} \frac{1+\frac{\omega_{E}}{2 t}}{\left(1+\frac{\omega_{E}}{4 t}\right)^{3 / 2}} .
$$

or the effective mass, defined as

$$
m / m^{*}=\left.\frac{1}{2 t} \frac{\partial^{2} E(k)}{\partial k^{2}}\right|_{k=0} .
$$

For a momentum independent self energy (as in the second order weak coupling expansion) these are simply related: $m^{*} / m=1 / z_{0}$. The residue clearly approaches the non-interacting value, unity, as $\omega_{E} \rightarrow \infty$, while it diverges as $\omega_{E} \rightarrow 0$. This indicates a breakdown in (weak coupling) perturbation theory in this limit, which is consistent with the fact established in Ref. [7] that the electron is polaron-like for all coupling strengths, i.e. there is an abrupt change in character at $g=0$. In fact, as established in Ref. [1] for a two-site model, and in Ref. 7], the effective mass diverges in the adiabatic limit for all coupling strengths (in 1D), a limit which we now approach numerically in Fig. 2b.

In two dimensions, as mentioned in Section II, we use $\lambda=g^{2} \omega_{E} /(2 \pi t)$. This actually uses the electron density of states at the bottom of the band, $N(-4 t)=1 /(4 \pi t)$, instead of the average density of states that is commonly used, $N_{\text {ave }}(0)=1 /(8 t)$. The reason for this choice is that we are studying the one electron sector, so the most pertinent density of states is the one at the bottom.

The self energy (in two dimensions (2D)) in weak coupling is given by

$$
\Sigma_{2 \mathrm{D}}(\omega+i \delta)=\frac{\lambda}{2} \frac{8 t \omega_{E}}{\omega-\omega_{E}} K\left[\left(\frac{4 t}{\omega-\omega_{E}}\right)^{2}\right],
$$

where $K(x) \equiv \int_{0}^{\pi / 2} d \theta \frac{1}{\sqrt{1-x \sin ^{2} \theta}}$ is the complete Elliptic integral of the first kind. This leads to a ground state energy, which, in weak coupling, is:

$$
E_{0}=-4 t\left(1+\frac{\lambda}{4} \frac{\omega_{E}}{t} \frac{1}{1+\omega_{E} /(4 t)} K\left[\frac{1}{\left(1+\omega_{E} /(4 t)\right)^{2}}\right]\right) .
$$

We can take the derivative of Eq. (9) to obtain:

$$
m^{*} / m=1+\frac{\lambda}{2} \frac{1}{1+\omega_{E} /(8 t)} E\left[\frac{1}{\left(1+\omega_{E} /(4 t)\right)^{2}}\right],
$$

where $E(x) \equiv \int_{0}^{\pi / 2} d \theta \sqrt{1-x \sin ^{2} \theta}$ is the complete Elliptic integral of the second kind. We have used, $\frac{\partial K(x)}{\partial x}=$ $\frac{1}{2 x}\left(\frac{E(x)}{1-x}-K(x)\right)$.

More familiar expressions are available, for cases when the arguments of the complete elliptic integrals are close to unity. This occurs for $\omega_{E}<<t$. Using $\lim _{m \rightarrow 1} K(m)=\frac{1}{2} \ln \left(16 / m_{1}\right) \stackrel{16}{,}$ where $m_{1}=1-m$, an approximate form for the ground state energy is:

$E_{0} \approx-4 t\left(1+\frac{\lambda}{4} \frac{\omega_{E}}{t} \frac{1}{1+\omega_{E} /(4 t)} \ln \left[4 \sqrt{\frac{2 t}{\omega_{E}}} \frac{1+\omega_{E} /(4 t)}{\sqrt{1+\omega_{E} /(8 t)}}\right]\right)$.

From Eq. (11) we obtain:

$m^{*} / m=1+\frac{\lambda}{2}+\lambda \frac{\omega_{E}}{16 t} \ln \left(\frac{32 t}{\omega_{E}}\right)+O\left(\left(\frac{\omega_{E}}{t}\right)^{2} \ln \left(\frac{t}{\omega_{E}}\right)\right)$.

Note that as $\omega_{E} / t \rightarrow 0$, the ground state energy approaches the non-interacting value, while the result for the effective mass approaches the one derived in the continuum limit by Cappelluti et al $\stackrel{17}{\underline{17}}$, and the mass enhancement is half that expected when $E_{F}$ is large.

In Fig.3a, we show the ground state energy of the 2D Holstein model as a function of $\lambda$ for a variety of phonon frequencies; we also show the result in the adiabatic limit as $\omega_{E} / t \rightarrow 0$. For the latter case, we adopted the iterative method described in Ref. [7,9], and used Lanczos diagonalization for the electronic portion. The abrupt transition occurs because we do not assume Bloch's theorem, and translational invariance is broken for sufficiently strong coupling. For non-zero phonon frequency we note the trend that as $\omega_{E} \rightarrow 0$, the crossover from free-electron-like behaviour to polaronic behaviour becomes more abrupt, though it is always smooth. 15 .

In Fig. 3b we show an expanded region in the weak coupling regime, where the perturbation theory results are also plotted. Note that they are quite accurate for all frequencies shown.

In Fig. 4a, we show the electron effective mass for the same parameters as in Fig. 3. In strong coupling the effective mass grows rapidly with coupling strength, as shown. However, this increase is even more pronounced as the phonon frequency decreases, until, as the adiabatic limit is approached, the increase becomes very nearly abrupt above a 'critical' coupling strength, as determined through the adiabatic calculation. At weak coupling, the effective mass is unity for large $\omega_{E}$ (not shown). As the phonon frequency decreases, the effective mass grows; however, for smaller phonon frequencies the effective mass will decrease again as the phonon frequency decreases (as can be seen from the cases shown). Both of these trends conspire to make the crossover more abrupt as the phonon frequency approaches zero.

In Fig. 4b we show an expanded region in the weak coupling regime (no $\log$ scale), where the perturbation theory results are also plotted. The results are certainly not as accurate as the ground state energy; however, the inversion with phonon frequency noted above is clearly obtained. 

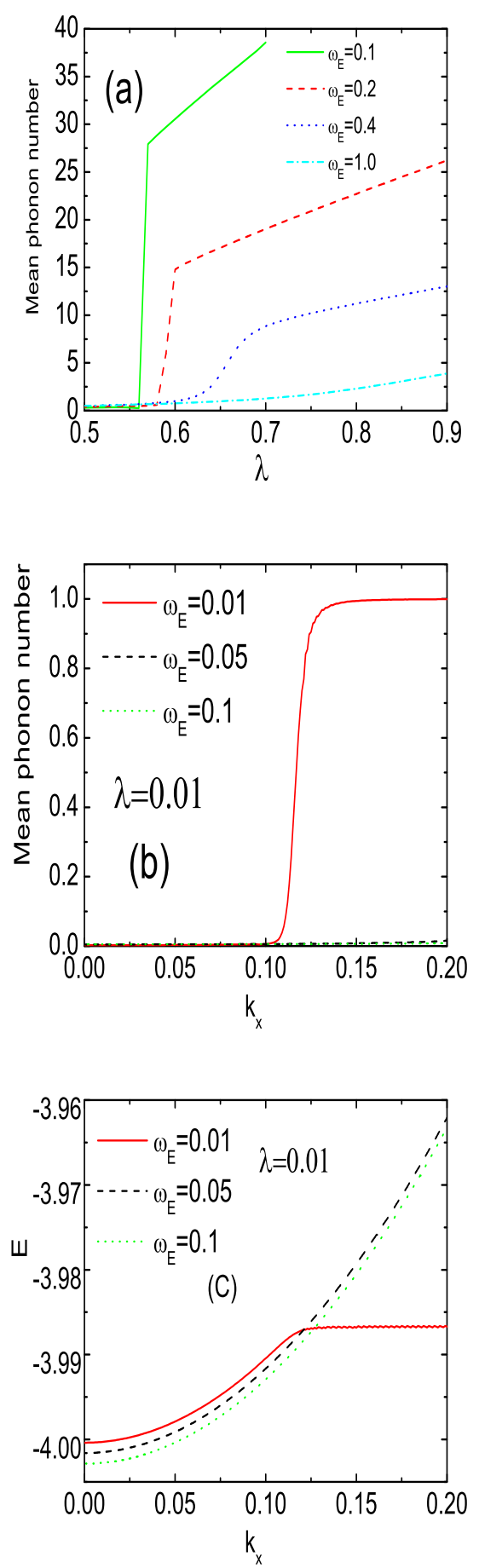

FIG. 5: (color online) (a) Mean phonon number vs. coupling strength for various phonon frequencies (in 2D). Note the increasingly abrupt behaviour as the phonon frequency decreases.

(b) Mean phonon number as a function of wave vector. Even for very small coupling strength there is an abrupt increase when the phonon frequency is small enough. Explanation is provided in the text, and is confirmed by (c) where the energy as a function of wave vector is plotted for the same parameters as in (b).

\section{MEAN PHONON NUMBER AND DISPERSION ANOMALIES}

Finally, we briefly examine the expectation of the number of phonons in the ground state, and the impact on the electronic dispersion relation. Restricting ourselves to two dimensions, we plot, in Fig.5a, the mean phonon number vs. coupling strength in the intermediate coupling regime for several phonon frequencies. The same trend as seen in Fig. 4 is apparent — beyond a 'special' coupling strength the mean phonon number grows very abruptly from near zero to some value, $N_{s}$, after which it continues to grow gradually as the coupling strength increases. The actual value of $N_{s}$ is close to the central value of the Poisson distribution as predicted by strong coupling perturbation theory ${ }^{9}$.

In Fig. 5b, we shows numerical results of the mean phonon number as a function of total momentum $k_{x}$, of the electron-phonon system, for a few values of phonon frequency, and for a very low value of coupling (so that the results are well converged). Despite this small value of coupling, convergence is difficult because we use $\omega_{E}$ as small as 0.01t. We apply our self-adaptive Lanczos method by first converging the results for some high momentum (say, $k_{x}=0.3$ - we keep $k_{y}=0$ ), and then lower the value of $k_{x}$ in small increments, and converge the calculation at each step, until we finally reach the desired end-point $\left(k_{x}=0\right)$. As Fig. 5b illustrates, for sufficiently small phonon frequency, the mean phonon number shows a sharp increase from close to zero to nearly unity at some wave-vector, say $k_{c}$. The reason for this is that the energy difference with the ground state will eventually exceed a value of order $\omega_{E}$; at this point it becomes energetically more favourable to use the zero momentum state (with much lower energy), and simply excite a phonon with the required momentum. Confirmation of this explanation is provided in Fig. 5(c), where the dispersion flattens abruptly beyond $k_{c}$, when the energy exceeds that of the ground state by an amount approximately equal to $\omega_{E}$. It retains this value because phonon momenta of any value are available with the same energy.

\section{SUMMARY}

We have implemented an adaptation to the variational method first suggested by Trugman, specifically to handle the adiabatic regime. In strong coupling our starting point leads to immediate convergence, while in the intermediate coupling regime a 'stepping-down' procedure allows for good convergence. Even in weak coupling, if the phonon frequency is significantly lower than the hopping parameter, our adaptive method is helpful, if not necessary.

By determining ground state properties as a function of decreasing phonon frequency we have established a connection between numerical results at small but nonzero phonon frequency, and adiabatic limit results ob- 
tained by using a semi-classical iterative procedure. It is clear that in one dimension no weak coupling perturbation regime exists, while in two dimensions (and higher) a definite weak coupling regime exists, and results derived within perturbation theory agree well with numerical results down to very low frequencies. Finally, as the phonon frequency decreases, more and more phonons are present in the ground state wave function, and these lead to anomalies in the electron dispersion relation.

It is somewhat ironic that the most significant polaronic effects occur in the adiabatic regime, as $\omega_{E} / t \rightarrow 0$. This is where weak coupling perturbation theory breaks down completely. The second order result, which is simply the so-called non-crossing approximation, fails to capture the rapid onset of multi-phonon excitations that form an integral part of the ground state wave function, as exemplified, for example, in Eq. (44); this is a breakdown that, for example, Alexandrov ${ }^{19}$ has repeatedly emphasized. At the same time, the so-called Migdal approximation $^{18}$, so key to the Eliashberg theory of superconductivity, is valid only in this limit. One then requires an understanding of how polaronic effects become minimized as more and more electrons are included in the problem. Apparently Pauli blocking plays an important role in mitigating the multi-phonon processes that constitute a single polaron. Future work will attempt to investigate this cross-over from polaron to weak coupling behaviour.

\section{Acknowledgments}

This work was supported in part by the Natural Sciences and Engineering Research Council of Canada (NSERC), by ICORE (Alberta), by Alberta Ingenuity, and by the Canadian Institute for Advanced Research (CIfAR). We thank Stuart Trugman for helpful correspondence in the early part of this work. FM is grateful to the Aspen Center for Physics, where some of this work was done (summer, 2007).

1 present address: Dept. of Oncology, University of Alberta, Edmonton, AB, Canada T6G 1 Z2

2 present address: Dept. of Mathematics, University of British Columbia, Vancouver, BC, Canada
1 T. Holstein, Ann. Phys. (New York) 8, 325 (1959).

2 J. Hubbard, Proc. Roy. Soc. A 276, 238 (1963); ibid., 277, 237 (1964).

${ }^{3}$ H. Fehske and S.A. Trugman, in Polarons in Advanced Materials edited by A. S. Alexandrov, Springer Series in Material Sciences 103 pp. 393-461, Springer Verlag, Dordrecht (2007).

4 A.S. Alexandrov, in Polarons in Advanced Materials, edited by A.S. Alexandrov, Springer Series in Materials Science, 103, pp. 257-310, Springer Verlag, Dordrecht (2007).

5 S.A. Trugman, in Applications of Statistical and Field Theory Methods to Condensed Matter, edited by D. Baeriswyl, A.R. Bishop, and J. Carmelo (Plenum Press, New York, 1990).

6 J. Bonča, S.A. Trugman, and I. Batistíc, Phys. Rev. B60, 1633 (1999).

7 V.V. Kabanov and O.Yu Mashtakov, Phys. Rev. B47, 6060 (1993).

8 A.S. Alexandrov, V.V. Kabanov, and D.K. Ray, Phys. Rev. B49, 9915 (1994).

9 F. Marsiglio, Physica C244 21, (1995).

10 L-C. Ku, S.A. Trugman, and J. Bonča, Phys. Reb. B65, 174306 (2002).
11 I.G. Lang and Yu. A. Firsov, Sov. Phys. JETP16, 1301 (1963); Sov. Phys. Solid State 52049 (1964).

12 A preliminary report of this work was given in Z. Li, D. Baillie, C. Blois, and F. Marsiglio, Bull. Am. Phys. Soc. 54, H13.9 (2009).

13 In the implementation of Trugman's method the use of a Bloch wave is implicit; nonetheless we include it explicitly in Eq. (4). Furthermore, in this representation we utilize phonon number states to reconstruct the exponential up to some high cutoff.

14 Two other possibilities for improving the original Trugman method, which we learned about after this work was completed, can be found in J. Bonča et al., Phys. Rev. B77, 054519 (2008), and A. Alvermann et al. arXiv:1001.2482.

15 H. Löwen, Phys. Rev. B37, 8661 (1988).

16 M. Abramowitz and I.A. Stegun, Handbook of Mathematical Functions (Dover, New York, 1972).

17 E. Cappelluti, C. Grimaldi, and F. Marsiglio, Phys. Rev. B76, 085334 (2007).

18 A.B.Migdal, Zh. Eksp. Teor.Fiz. 34, 1438 (1958) [Sov. Phys. JETP 7, 996 (1958)].

19 A.S. Alexandrov, Europhys. Lett. 56, 92 (2001). 